\title{
HLA-DR (la) Immune Phenotype Predicts Outcome for Patients with Diffuse Large Cell Lymphoma
}

\author{
Thomas P. Miller, Scott M. Lippman, Catherine M. Spier, Donald J. Slymen, and Thomas M. Grogan \\ Departments of Medicine and Pathology, and the Arizona Cancer Center, University of Arizona, Tucson, Arizona 85724
}

\begin{abstract}
The clinical utility for establishing the immune phenotype in patients with non-Hodgkin's lymphoma is controversial. To help resolve this dilemma, we studied 104 consecutive patients with diffuse large cell lymphoma, the most common subtype of potentially curable non-Hodgkin's lymphomas. The presence or absence of the human class II histocompatibility antigen was determined using the monoclonal antibody anti-HLA-DR (Ia), and the results correlated with pretreatment clinical features and survival. We found that eight HLA-DR negative patients had similar pretreatment clinical characteristics compared with 96 HLA-DR positive patients, but HLA-DR negative patients had a significantly shorter survival duration compared with HLA-DR positive patients $(P=0.003$ log-rank). The median survival of the HLA-DR negative patients was 0.5 years compared to $2.8 \mathrm{yr}$ for the HLA-DR positive patients. No HLA-DR negative patient survived beyond 1.5 yr. A multivariate analysis, adjusting for prognostic factors of known clinical significance, confirmed the importance of HLA-DR as a prognostic factor $(P=0.016)$. We conclude that determining the presence of HLA-DR is a relatively simple pretreatment study that identifies a small but important group of patients who are not curable using currently available combination chemotherapy.
\end{abstract}

\section{Introduction}

All patients with diffuse large cell lymphoma (DLCL), ${ }^{1}$ the most common subtype of intermediate grade non-Hodgkin's lymphoma (NHL) (1), currently receive the same initial chemotherapy resulting in cure for $30 \%$ and eventual relapse and death for $70 \%$ (2). Although patients with DLCL appear histologically similar, the disease is heterogeneous with regard to clinical course and survival outcome. In an effort to more accurately predict outcome prior to treatment for patients with

Address reprint requests to Dr. Miller, Arizona Cancer Center, $1501 \mathrm{~N}$. Campbell Ave., Tucson, AZ 85724.

Received for publication 7 March 1988.

1. Abbreviations used in this paper: CVP, cyclophosphamide, vincristine, and prednisone; DAB, diaminobenzidine tetrahydrochloride; DLCL, diffuse large cell lymphoma; LFA-1, lymphocyte function-associated antigen; NHL, non-Hodgkin's lymphoma.

J. Clin. Invest.

(c) The American Society for Clinical Investigation, Inc.

0021-9738/88/07/0370/03 \$2.00

Volume 82, July 1988, 370-372
DLCL, investigators have explored the potential prognostic value of determining the immune phenotype of these NHLs. The results of those efforts are inconclusive with some studies showing no prognostic value (3-5) and others suggesting utility for determining the immune phenotype of DLCL (6-8). These disparate results may be related to any of several problems of past study designs including; small numbers of patients studied, comparisons between heterogenous histologic subgroups, and selection of monoclonal antibodies having little biological significance.

In 1987, Clayberger et al. demonstrated a correlation between loss of lymphocyte-function-associated antigen (LFA-1) and histologic grading of NHL (9). Furthermore, patients lacking LFA-1 seemed to relapse and fail therapy more often than patients expressing LFA-1. The authors speculated that the selective loss of molecules involved in the process of cellcell interaction might allow the disruption of the immune network and expansion of the malignant cells. To test that hypothesis, we chose to study HLA-DR (Ia), a class II human histocompatibility antigen, also thought to be essential for cell-cell interactions (10). In an attempt to obviate some of the problems of past reports and to further explore the significance of antigens relevant to cell-cell interaction, we studied 104 consecutive patients with a single morphologic subtype of NHL (DLCL) treated over a 10-year period and found that the absence of HLA-DR (Ia) identifies a group of patients with an extremely short survival duration who do not benefit from currently available potentially curative chemotherapy.

\section{Methods}

From 1978 to 1987,115 consecutive patients with the initial diagnosis of DLCL were prospectively accrued on study and evaluated at the Arizona Cancer Center. Our analyses exclude 10 patients whose tissue specimens were inadequate for detailed immunologic study, and 1 patient who had an indeterminate result, leaving 104 patients for study. We used a previously described immunohistochemical method (6) employing primary mouse antihuman monoclonal antibodies, biotin conjugated second stage, avidin-horseradish peroxidase, and the chromagen diaminobenzidine tetrahydrochloride (DAB) as a detection agent on snap-frozen tissue sections. We employed the monoclonal antibody anti-HLA-DR (Becton Dickinson, Mountain View, CA) to detect the human class II histocompatibility antigen, HLA-DR (Ia). The extent of disease was determined using pretreatment clinical data obtained from physical examination, routine blood counts and chemistries, bone marrow biopsy, chest radiograph, and either a computed tomography (CT) scan of the abdomen or lymphangiogram. Patients were assigned a clinical stage according to the Ann Arbor system (11).

We defined a complete remission as the disappearance of all disease that was present at the initiation of treatment. Survival was calculated 
from the date of diagnosis to the date of death (any cause) or last contact. Survival curves were estimated by the Kaplan and Meier method, and comparisons between curves were based on the log-rank and Gehan's generalized Wilcoxon tests (12). Cox's proportional hazards model was used to assess the effect of HLA-DR on survival after adjusting for relevant prognostic factors (12). All tests were two tailed. All patients are included in the survival analysis regardless of the treatment received or cause of death.

Treatment consisted of potentially curative doxorubicin-containing combination chemotherapy in $84 \%$ of patients as previously detailed (13) including the CHOP regimen in 55\%, CHOP plus irradiation in $15 \%$, ProMACE-CytaBOM in 6\%, MACOP-B in 5\%, and $\mathrm{m}-$ BACOD in $4 \% .16 \%$ of patients received palliative treatment including CVP (cyclophosphamide, vincristine, and prednisone) in $4 \%$, irradiation alone in $4 \%$, surgery followed by irradiation in $2 \%$, and no therapy in $5 \%$.

\section{Results}

The clinical characteristics of the 104 patients are summarized in Table I. In general, the clinical variables are similar between 96 HLA-DR positive patients and 8 HLA-DR negative patients. We note some differences including an older median age for the HLA-DR negative group compared to the HLADR positive group (69.3 yr vs. $56.3 \mathrm{yr}$, respectively), a lower proportion of males in the HLA-DR negative group (62.5 vs. $37.5 \%$ ), and a higher frequency of systemic symptoms (B symptoms) in the HLA-DR negative group (62.5 versus $41.7 \%$ ). Importantly, the proportion of patients receiving curative treatment (doxorubicin-containing multiagent chemotherapy) and the proportion achieving a complete remission are similar.

Survival was significantly different between the two groups (Fig. 1, log-rank $P=0.003$, Wilcoxon $P=0.006$ ). The median survival of the HLA-DR negative patients was $0.5 \mathrm{yr}$ compared to $2.8 \mathrm{yr}$ for the HLA-DR positive patients. No HLA-

Table I. Clinical Characteristics of 104 Patients with Diffuse Large Cell Non-Hodgkin's Lymphoma (HLA-DR Positive Versus HLA-DR Negative Phenotypes)

\begin{tabular}{|c|c|c|c|c|}
\hline \multirow[b]{2}{*}{ Characteristic } & \multicolumn{2}{|c|}{$\begin{array}{c}\text { HLA-DR (la) } \\
\text { Positive }(n=96) \\
\end{array}$} & \multicolumn{2}{|c|}{$\begin{array}{c}\text { HLA-DR (la) } \\
\text { Negative }(n=8) \\
\end{array}$} \\
\hline & No. & (\%) & No. & $(\%)$ \\
\hline $\begin{array}{l}\text { Age: Median } \\
\text { range }\end{array}$ & \multicolumn{2}{|c|}{$\begin{array}{l}56.3 \mathrm{yr} \\
{[12-86]}\end{array}$} & \multicolumn{2}{|c|}{$\begin{array}{l}69.3 \mathrm{yr} \\
{[20-73]}\end{array}$} \\
\hline Age: $\geq 65 \mathrm{yr}$ & 33 & $(34.4)$ & 5 & $(62.5)$ \\
\hline Sex: Males & 60 & $(62.5)$ & 3 & (37.5) \\
\hline $\begin{array}{l}\text { Stage I or II } \\
\text { III or IV }\end{array}$ & $\begin{array}{l}29 \\
67\end{array}$ & $\begin{array}{l}(30.2) \\
(69.8)\end{array}$ & $\begin{array}{l}2 \\
6\end{array}$ & $\begin{array}{l}(25.0) \\
(75.0)\end{array}$ \\
\hline B symptoms & 40 & $(41.7)$ & 5 & $(62.5)$ \\
\hline Tumor bulk: $\geq 10 \mathrm{~cm}$ & 20 & $(21.3)$ & 2 & $(25.0)$ \\
\hline LDH: $\leq 225$ & 39 & $(60.0)$ & 3 & $(60.0)$ \\
\hline$>225$ & 26 & $(40.0)$ & 2 & $(40.0)$ \\
\hline Not performed & 31 & - & 3 & - \\
\hline Extranodal disease & 82 & $(85.4)$ & 6 & $(75.0)$ \\
\hline Curative treatment & 80 & $(83.3)$ & 7 & $(87.5)$ \\
\hline Complete response & 56 & $(60.2)$ & 5 & $(62.5)$ \\
\hline
\end{tabular}

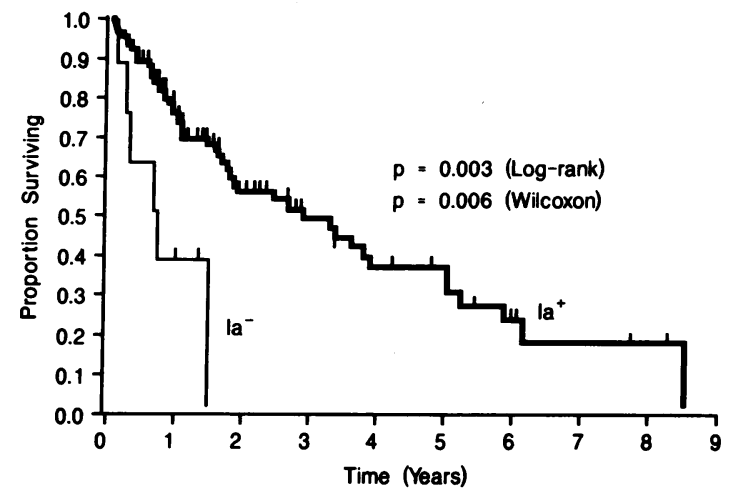

Figure 1. Survival for 104 consecutive patients with diffuse large cell lymphoma comparing 96 HLA-DR positive patients $(\mathrm{Ia}+)$ to 8 HLA-DR negative patients (Ia-).

DR negative patient has survived beyond 1.5 yr. Because B symptoms and age are known predictors of survival, both factors were included in a multivariate analysis with HLA-DR. After adjustment, HLA-DR remained a major independent prognostic factor for survival $(P=0.016)$.

\section{Discussion}

This study of a single histologic subtype of NHL suggests that patients with an immune phenotype that lacks the HLA-DR (Ia) antigen comprise a subgroup who are not curable using currently available combination chemotherapy. In fact, the survival estimates of the HLA-DR negative patients appear worse than those achieved using radiotherapy alone as reported by Jones et al. in 1973 before the availability of potentially curative combination chemotherapy (14).

Preliminary results from two other groups seem to support this finding $(15,16)$. Kluin et al. noted an adverse effect on survival for 6 HLA-DR negative patients compared with 129 HLA-DR positive patients having NHL of B-cell lineage including all 10 Working Formulation histologic subtypes (15). In that study the median survival for HLA-DR negative patients was 5 mo. Hart et al. noted that the presence of HLADR conferred a significant survival advantage on patients among 106 patients with DLCL $(P<0.01)(16)$. In this latter study, the number of HLA-DR negative patients and the median survival were not noted.

The utility of establishing the immune phenotype for patients with NHL remains controversial. Most prior studies have explored the importance of determining the B-cell versus $T$-cell groups or the presence versus absence of surface immunoglobulins (3-8). More recent trials have explored additional surface and nuclear antigens using newer monoclonal antibodies with some success including the activation factors, transferrin receptor (17), and HLA-DR $(15,16)$, and proliferation antigens such as $\mathrm{Ki}-67(18,19)$. These recent studies, together with the current report, present a cogent argument to prospectively test the prognostic value of the immune phenotype in NHL including adequate numbers of patients with a single histologic subtype. Furthermore, the current study supports the hypothesis that cell-cell interactions may be an essential prerequisite for cure of NHL and the loss of either LFA-1 (9) or HLA-DR may impair cytotoxic tumor infiltrating lymphocyte response (10). In that circumstance, successful treatment 
outcome may necessitate restoration of tumor antigenicity and tumor histocompatibility antigen expression. Since alpha and gamma interferons are known to stimulate HLA-DR expression (20), restoration of antigenicity may be effected with those cytokines, and future treatment strategies may require the addition of cytokines to chemotherapy.

\section{Acknowledgments}

Supported in part by Public Health Services grants CA-17094 and CA-23074 from the National Cancer Institute, National Institutes of Health, Department of Health and Human Services.

\section{References}

1. The Non-Hodgkin's Lymphoma Pathologic Classification Project. 1982. National Cancer Institute sponsored study of classifications of non-Hodgkin's lymphomas. Summary and description of a working formulation for clinical usage. Cancer. 49:2112-2135.

2. Coltman, C. A., S. E. Dahlberg, S. E. Jones, T. P. Miller, B. W. Dana, E. M. McKelvey, R. J. Hartsock, and D. O. Dixon. 1986. CHOP is curative in thirty percent of patients with large cell lymphoma. A twelve year Southwest Oncology Group follow-up. Proc. Am. Soc. Clin. Oncol. 5:197. (Abstr.)

3. Cossman, J., E. S. Jaffe, and R. I. Fisher. 1984. Immunologic phenotypes of diffuse, aggressive non-Hodgkin's lymphomas. Correlation with clinical features. Cancer (Phila.). 54:1310-1317.

4. Levine, A. M., C. R. Taylor, D. R. Schneider, S. C. Koehler, S. J. Forman, A. Lichtenstein, R. J. Lukes, and D. I. Feinstein. 1981. Immunoblastic sarcoma of $\mathrm{T}$-cell versus B-cell origin. I. Clinical features. Blood. 58:52-61.

5. Horning, S. J., L. M. Weiss, G. S. Crabtree, and R. A. Warnke. 1986. Clinical and phenotypic diversity of T cell lymphomas. Blood. 67:1578-1582.

6. Grogan, T. M., K. Fielder, C. Rangel, C. Jolley, D. Wirt, M. J. Hicks, T. P. Miller, R. Brooks, G. Greenberg, and S. E. Jones. 1985. Peripheral T-cell lymphoma: Aggressive disease with heterogeneous immunotypes. Am. J. Clin. Pathol. 83:279-288.

7. Greer, J. P., J. C. York, J. B. Cousar, R. T. Mitchell, J. M. Flexner, R. D. Collins, and R. S. Stein. 1984. Peripheral T-cell lymphoma: A clinicopathologic study of 42 cases. J. Clin. Oncol. 2:788798.

8. Brouet, J. C., C. Rabian, C. Gisselbrecht, and G. Flandrin. 1984. Clinical and immunologic study of non-Hodgkin T-cell lymphomas (cutaneous and lymphoblastic lymphomas excluded). Br. J. Haematol. 57:315-327.

9. Clayberger, C., A. Wright, L. J. Medeiros, T. D. Koller, M. P. Link, S. D. Smith, R. A. Warnke, A. M. Krensky. 1987. Absence of cell surface LFA-1 as a mechanism of escape from immunosurveillance. Lancet. ii:533-536.

10. Jacob F. 1977. Mouse teratocarcinoma and embryonic antigens. Immunol. Rev. 33:3-47.

11. Carbone, P. P., H. S. Kaplan, K. Musshoff, D. W. Smithers, and M. Tubiana. 1977. Report of the committee on Hodgkin's disease staging classification. Cancer Res. 31:1860-1861.

12. Cox, D. R., and D. Oakes. 1984. Analysis of Survival Data. Chapman and Hall, London. 201 pp.

13. Fisher, R. I., T. P. Miller, B. W. Dana, S. E. Jones, S. Dahlberg, and C. A. Coltman. 1987. Southwest Oncology Group clinical trials for intermediate and high-grade non-Hodgkin's lymphomas. Semin. $\mathrm{He}$ matol. 24(Suppl.):21-25.

14. Jones, S. E., Z. Fuks, M. Bull, M. E. Kadin, R. F. Dorfman, H. S. Kaplan, S. A. Rosenberg, and K. Hun. 1973. Non-Hodgkin's lymphoma IV. Clinicopathologic correlation in 405 cases. Cancer Phila. 31:806-823.

15. Kluin, P., K. v. Gromingen, M. v.d. Sandt, P. Spaander, J. Te Velde, H. Haak, T. Stijnen, and R. Otter. 1987. Histoimmunopathology related to survival in a regional registry of non-Hodgkin's lymphomas. Program and Abstracts of the Third International Conference on Malignant Lymphoma, Lugano, Switzerland. 125 (Abstr.)

16. Hart, S., P. Toghill, G. Vaughan-Hudson, and K. A. MacLennan. 1987. Phenotypic analysis of diffuse large cell lymphoma in paraffin sections: The relationship to prognosis and natural history. Program and Abstracts of the Third International Conference on Malignant Lymphoma, Lugano, Switzerland, June. 81 (Abstr.)

17. Habeshaw, J. A., T. A. Lister, and A. G. Stansfeld. 1983. Correlation of transferrin receptor expression with histologic class and outcome in non-Hodgkin's lymphoma. Lancet. i:498-501.

18. Gerdes, J., H. Stein, S. Pileri, M. T. Rivano, M. Gobbi, E. Ralfkiaer, K. M. Nielsen, G. Pallesen, H. Bartels, G. Palestro, and G. Dilsol. 1987. Prognostic relevance of tumour-cell growth fraction in malignant non-Hodgkin's lymphomas. Lancet. ii:448-449.

19. Grogan, T. M., S. M. Lippman, C. M. Spier, D. J. Slymen, J. A. Rybski, C. S. Rangel, L. C. Richter, and T. P. Miller. 1988. Independent prognostic significance of a nuclear proliferation antigen in diffuse large cell lymphomas as determined by the monoclonal antibody Ki-67. Blood. 71:1157-1160.

20. Greiner, J. W., P. Horan Hand, D. Colcher, M. Weeks, A. Thor, P. Noguchi, S. Pestka, and J. Schlom. 1987. Modulation of human tumor antigen expression. J. Lab. Clin. Med. 109:244-261. 